() А.Н. Казанцев ${ }^{1 *}$, Р.А. Виноградов ${ }^{2,3}$, М.А. Чернявский ${ }^{4}$, В.Н. Кравчук ${ }^{5,6}$, Д.В. Шматов ${ }^{7}$, А.А. Сорокин ${ }^{7}$, А.А. Ерофеев ${ }^{8}$, В.А. Луценко ${ }^{9}$, Р.В. Султанов ${ }^{9}$, А.Р. Шабаев ${ }^{10}$, И.М. Раджабов ${ }^{11}$, Г.Ш. Багдавадзе ${ }^{6}$, Н.Э. Заркуа ${ }^{6}$, В.В. Матусевич²,

Е.Ф. Вайман ${ }^{12}$, А.И. Солобуев ${ }^{12}$, С.В. Артюхов ${ }^{1}$, Р.Ю. Лидер ${ }^{12}$, В.А. Порханов ${ }^{2}$, Г.Г. Хубулава ${ }^{5,13}$

'Городская Александровская больница, Санкт-Петербург

${ }^{2}$ Краевая клиническая больница №1 им. проф. С.В. Очаповского, Краснодар

${ }^{3}$ Кубанский государственный медицинский университет, Краснодар

${ }^{4}$ Национальный медицинский исследовательский центр им. В.А. Алмазова, Санкт-Петербург

${ }^{5}$ Военно-медицинская академия им. С.М. Кирова, Санкт-Петербург

${ }^{6}$ Северо-Западный государственный медицинский университет им. И.И. Мечникова, Санкт-Петербург

${ }^{7}$ Клиника высоких медицинских технологий им. Н.И. Пирогова, Санкт-Петербург

${ }^{8}$ Городская многопрофильная больница №2, Санкт-Петербург

${ }^{9}$ Кемеровская областная клиническая больница им. С.В. Беляева, Кемерово

${ }^{10}$ Кемеровский областной клинический кардиологический диспансер им. акад. Л.С. Барбараш, Кемерово

${ }^{11}$ Главный военный клинический госпиталь им. акад. Н.Н. Бурденко, Москва

${ }^{12}$ Кемеровский государственный медицинский университет, Кемерово

${ }^{13}$ Первый Санкт-Петербургский государственный медицинский университет им. акад. И.П. Павлова, Санкт-Петербург

ОБОСНОВАНИЕ. Сахарный диабет (СД) 2 типа является одним из важных маркеров развития неблагоприятных кардиоваскулярных событий после каротидной эндартерэктомии (КЭЭ). Однако исследования по данному вопросу построены на небольших выборках больных и не учитывают вид операции как дополнительный фактор потенциально негативного влияния на течение послеоперационного периода.

ЦЕЛЬ. Анализ госпитальных и отдаленных результатов эверсионной и классической КЭЭ с пластикой зоны реконструкции биологической заплатой у пациентов с СД 2 типа (СД2) и без него.

МАТЕРИАЛЫ И МЕТОДЫ. В данное многоцентровое ретроспективное исследование за период с января 2010 по декабрь 2020 гг. включен 5731 пациент. В зависимости от наличия/отсутствия СД2 и вида реализованной КЭЭ было сформировано 4 группы: 1-я группа - 12,2\% (n=702) - пациенты с СД2 после эверсионной КЭЭ; 2-я группа 55,0\% (n=3153) - пациенты без СД2 после эверсионной КЭЭ; 3-я группа - 8,5\% (n=484) - пациенты с СД2 после классической КЭЭ; 4-я группа - 24,3\% (n=1392) - пациенты без СД2 после классической КЭЭ. Длительность послео-

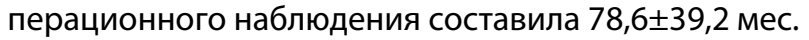

РЕзУЛЬтАТЫ. На отдаленном этапе наблюдения пациенты с СД2 после классической техники операции продемонстрировали наибольшие показатели всех вариантов осложнений: смерть ( $<<0,0001)$, инфаркт миокарда ( ишемический инсульт ( $<<0,0001)$, рестеноз/окклюзия внутренних сонных артерий (BCA) (p<0,0001), комбинированная конечная точка ( $<<0,0001)$. При этом вторую позицию по распространенности неблагоприятных событий заняла группа эверсионной КЭЭ с нарушением углеводного обмена. Такие обстоятельства демонстрируют, что имплантация заплаты сопровождается повышенным риском развития не только инфаркта миокарда, но и рестеноза зоны реконструкции, а также связанного с ним ишемического инсульта, что продемонстрировали наши результаты.

ЗАКЛЮЧЕНИЕ. Пациенты с СД2 и КЭЭ в анамнезе подвержены повышенному риску развития ишемического инсульта на госпитальном этапе наблюдения и всех неблагоприятных кардиоваскулярных состояний (летальный исход, инфаркт миокарда, ишемический инсульт, рестеноз или окклюзия ВСА в зоне реконструкции) в отдаленном послеоперационном периоде.

КЛЮЧЕВЫЕ СЛОВА: каротидная эндартерэктомия; сахарный диабет; классическая каротидная эндартерэктомия; эверсионная каротидная эндартерэктомия; рестеноз; заплата; биологическая заплата

\title{
RESULTS OF DIFFERENT KINDS OF CAROTID ENDARTERECTOMY IN PATIENTS WITH AND WITHOUT TYPE 2 DIABETES MELLITUS
}

(c) Anton N. Kazantsev ${ }^{*}$, Roman A. Vinogradov2,3, Michael A. Chernyavsky4, Vyacheslav N. Kravchuk ${ }^{5,6}$, Dmitriy V. Shmatov Andrey A. Sorokin ${ }^{7}$, Alexandr A. Erofeev ${ }^{8}$, Victor A. Lutsenko ${ }^{9}$, Roman V. Sultanov ${ }^{9}$, Amin R. Shabaev ${ }^{10}$, Islam M. Radjabov ${ }^{11}$, Goderzi Sh. Bagdavadze ${ }^{6}$, Nonna E. Zarkua ${ }^{6}$, Vyacheslav V. Matusevich², Evgeniy F. Vaiman ${ }^{12}$, Aleksey I. Solobuev ${ }^{12}$, Sergey V. Artyukhov' ${ }^{\text {, Roman Yu. Lider }}{ }^{12}$, Vladimir A. Porkhanov², Gennadiy G. Khubulava ${ }^{5,13}$ 
${ }^{1}$ City Alexandrovskaya Hospital, St. Petersburg, Russia

${ }^{2}$ Research Institute Regional Clinical Hospital No. 1 named. prof. S.V. Ochapovsky, Krasnodar, Russia

${ }^{3}$ Kuban State Medical University, Krasnodar, Russia

${ }^{4}$ National Medical Research Center named after V.A. Almazov, Saint Petersburg, Russia

${ }^{5}$ Military Medical Academy named after SM Kirov, Saint Petersburg, Russia

${ }^{6}$ North-Western State Medical University named after I.I. Mechnikov, Saint Petersburg, Russia

${ }^{7}$ Clinic of high medical technologies named after N.I. Pirogov, Saint Petersburg, Russia

${ }^{8}$ City Multidisciplinary Hospital No. 2, Saint Petersburg, Russia

${ }^{9}$ Kemerovo Regional Clinical Hospital named after S.V. Belyaeva, Kemerovo, Russia

${ }^{10}$ Kemerovo Regional Clinical Cardiological Dispensary named after acad. L.S. Barbarash, Kemerovo, Russia

${ }^{11}$ Main Military Clinical Hospital named after acad. N.N.Burdenko, Moscow, Russia

${ }^{12}$ Kemerovo State Medical University, Kemerovo, Russia

${ }^{13}$ First Saint Petersburg State Medical University named after I.I. acad. I.P. Pavlova, Saint Petersburg, Russia

BACKGROUND: Type 2 diabetes mellitus (DM) is one of the important markers for the development of adverse cardiovascular events after carotid endarterectomy (CEE). However, studies on this issue are based on small sample of patients and do not take into account the type of surgery as an additional factor of potentially negative impact on the course of the postoperative period.

AIM: Analysis of hospital and long-term results of eversion and classical CEE with plastic surgery of the reconstruction zone with a biological patch in patients with type 2 diabetes and without it.

MATERIALS AND METHODS: In this multicenter retrospective study from January 2010 to December 2020. included 5731 patients. Depending on the presence / absence of type 2 diabetes and the type of implemented CEE, 4 groups were formed: group $1-12.2 \%(n=702)$ - patients with type 2 diabetes and eversion CEE; Group $2-55.0 \%(n=3153)$ patients without type 2 diabetes and eversion CEE; Group $3-8.5 \%(n=484)$ patients with type 2 diabetes and classical CEE; Group $4-24.3 \%(n=1392)$ patients without type 2 diabetes and classical CEE. The duration of postoperative follow-up was $78.6 \pm 39.2$ months.

RESULTS: At the long-term follow-up stage, patients with type 2 diabetes after the classical surgical technique demonstrated the highest rates of all types of complications: death $(p<0.0001), M I(p=0.011)$, ischemic stroke $(p<0.0001)$, restenosis / occlusion of the ICA ( $<<0.0001)$, combined end point $(p<0.0001)$. At the same time, the group of eversion CEE with impaired carbohydrate metabolism took the second position in terms of the prevalence of adverse events. These circumstances demonstrate that patch implantation is accompanied by an increased risk of developing not only myocardial infarction, but also restenosis of the reconstruction zone, as well as the associated ischemic stroke, which was demonstrated by our results.

CONCLUSION: Patients with type 2 diabetes and a history of CEE are at increased risk of ischemic stroke at the hospital stage of observation and all unfavorable cardiovascular conditions (death, myocardial infarction, ischemic stroke, restenosis or ICA occlusion in the reconstruction zone) in the long-term postoperative period.

KEYWORDS: carotid endarterectomy; diabetes mellitus; classical carotid endarterectomy; eversion carotid endarterectomy; restenosis; patch; biological patch

Сахарный диабет 2 типа (СД2) всегда играл одну из ключевых ролей в прогрессировании каротидного атеросклероза [1, 2]. Известно, что комбинация в виде нарушения углеводного обмена и стенозов экстракраниальных артерий диагностируется у каждого третьего пациента с мультифокальным атеросклерозом (МФА), повышая риск развития острого нарушения мозгового кровообращения/транзиторной ишемической атаки (ОНМК/ТИА) до 74\% [3-5]. Золотым стандартом хирургического лечения больных с гемодинамически значимыми стенозами внутренних сонных артерий (ВСА) является каротидная эндартерэктомия (КЭЭ) [6, 7]. Существует два наиболее распространенных варианта этой операции - классическая с пластикой зоны реконструкции заплатой и эверсионная $[7,8]$. При классической КЭЭ производится продольная артериотомия общей сонной артерии с переходом на внутреннюю сонную артерию (ВСА). Затем открытым способом из просвета артерий удаляется атеросклеротическая бляшка. После этого производится пластика зоны реконструкции заплатой (биологической, синтетической, аутовенозной) [7, 8].

Эверсионная КЭЭ чаще всего выполняется по одной из методик, наиболее распространенные из которых в разные годы описали А.В. Покровский, А.Н. Казанцев, Р.А. Виноградов и др. [6, 8]. Техника всегда начинается с отсечения ВСА от устья. Далее атеросклеротическая бляшка из просвета артерии удаляется путем ее выворачивания. После этого ВСА имплантируется на прежнее место $[6,8]$.

Выбор техники операции по данным действующих рекомендаций осуществляется оперирующим хирургом [1-5]. При этом СД2 не рассматривается как противопоказание для того или иного вида КЭЭ [1-5].

По мнению ряда авторов, СД2 является одним из важных маркеров развития неблагоприятных кардиоваскулярных событий после КЭЭ $[9,10]$. В других работах доказан вклад данной патологии в формирование рестеноза ВСА $[11,12]$. Однако все эти исследования построены на небольших выборках больных и не учитывают вид операции как дополнительный фактор потенциально негативного 
влияния на течение послеоперационного периода. Само по себе наличие инородного материала - заплаты при классической КЭЭ - на фоне СД2, вероятно, может ассоциироваться с непредсказуемыми изменениями в зоне анастомоза с последующей активацией воспаления, гиперплазии неоинтимы, отторжением. Таким образом, является ли СД2 катализатором этих процессов при реализации различных видов КЭЭ, - остается неясным.

\section{ЦЕЛЬ ИССЛЕДОВАНИЯ}

Изучение госпитальных и отдаленных результатов эверсионной и классической КЭЭ с пластикой зоны реконструкции заплатой у пациентов с СД2 и без него.

\section{МАТЕРИАЛЫ И МЕТОДЫ}

\section{Место и время проведения исследования}

Место проведения. В данном исследовании приняло участие 13 учреждений: ГБУЗ «Городская Александровская больница», г. Санкт-Петербург; ГБУЗ «НИИ Краевая клиническая больница №1 им. проф. С.В. Очаповского», г. Краснодар; Кафедра хирургии №1 ФПК и ППС Кубанского государственного медицинского университета, г. Краснодар; ФГБУ «Национальный медицинский исследовательский центр им. В.А. Алмазова» Минздрава России, г. Санкт-Петербург; ФГБВОУ ВО «Военно-медицинская академия им. С.М. Кирова», г. Санкт-Петербург; ФГБОУ ВО «Северо-Западный государственный медицинский университет им. И.И. Мечникова», г. СанктПетербург; Клиника высоких медицинских технологий им. Н.И. Пирогова СПБГУ, г. Санкт-Петербург; ГБУЗ «Городская многопрофильная больница №2», г. СанктПетербург; ГАУЗ КО «Кемеровская областная клиническая больница» им. С.В. Беляева, г. Кемерово; ГБУз Кемеровской области «Кемеровский областной клинический кардиологический диспансер им. акад. Л.С. Барбараш», г. Кемерово; ФГБУ «Главный военный клинический госпиталь им. акад. Н.Н. Бурденко» Министерства обороны Российской Федерации, г. Москва; ФГБОУ ВО «Кемеровский государственный медицинский университет» Министерства здравоохранения Российской Федерации, г. Кемерово; Первый Санкт-Петербургский государственный медицинский университет им. акад. И.П. Павлова, г. Санкт-Петербург.

Время исследования. С января 2010 по декабрь 2020 гг.

Изучаемые популяции (одна или несколько)

В исследование включен 5731 пациент с гемодинамически значимыми стенозами ВСА. В зависимости от наличия/отсутствия СД2 и вида реализованной КЭЭ было сформировано 4 группы:

1-я группа - 12,2\% (n=702) - пациенты с СД2 после эверсионной КЭЭ;

2-я группа - 55,0\% (n=3153) - пациенты без СД2 после эверсионной КЭЭ;

3-я группа - 8,5\% (n=484) - пациенты с СД2 после классической КЭЭ;

4-я группа - 24,3\% (n=1392) - пациенты без СД2 после классической КЭЭ;

Критерии включения. Показания для КЭЭ согласно действующим рекомендациям.
Критерии исключения. Наличие СД2, установленного эндокринологом (в 1-й и 3-й группах); отсутствие патологии, лимитирующей наблюдение пациента в отдаленном периоде наблюдения (онкология и т.д.). Критерии исключения подразумевали показатели, не удовлетворяющие вышеизложенным пунктам включения.

Способ формирования выборки из изучаемой популяции (или нескольких выборок из нескольких

изучаемых популяций)

Выборка формировалась путем сплошного включения наблюдений.

\section{Дизайн исследования}

Настоящее исследование является многоцентровым обсервационным динамическим ретроспективным четырехвыборочным сравнительным контролируемым нерандомизированным. Минимальный срок между началом и окончанием наблюдения участников исследования составил 5 мес. Максимальный срок между началом и окончанием наблюдения участников исследования составил 120 мес.

\section{Методы}

Выбор стратегии реваскуляризации осуществлялся мультидисциплинарным консилиумом, включающим сердечно-сосудистого хирурга, эндоваскулярного хирурга, нейрохирурга, кардиолога, невролога, анестезиолога, реаниматолога, эндокринолога. При выполнении классической КЭЭ использовалась заплата из диэпоксиобработанного ксеноперикарда.

Для определения глюкозы натощак исследовалась плазма венозной крови. Тяжесть коронарного атеросклероза рассчитывалась при помощи интерактивного калькулятора SYNTAX Score (www.syntaxscore.com). По тяжести поражения на основании данного калькулятора выделяется следующая градация: низкий уровень поражения ( $\leq 22$ баллов), промежуточный (23-32 балла) и тяжелый ( $\geq 33$ баллов). Визуализация стенозов ВСА производилась при помощи цветного дуплексного сканирования с последующей ангиографией (АГ) или мультиспиральной компьютерной томографией с АГ (МСКТ АГ). Выраженность поражения оценивалась по классификации NASCET (https:/www.openneuro.ru/ doctors/cerebrovascular/carotid-stenoses).

Компенсаторные возможности церебрального кровотока во время КЭЭ оценивались следующим образом. При уровне систолического артериального давления (САД) $\leq 160$ мм рт.ст. выполнялось фармакологическое повышение артериального давления (АД) до 190/100 мм рт.ст. Затем внутривенно вводилось 5 тысяч ЕД гепарина, осуществлялось пережатие артерий. Производилось инвазивное измерение ретроградного давления во ВСА. При уровне АД менее $60 \%$ от системного применялся временный шунт (ВШ).

Под контрольными точками понималось развитие таких неблагоприятных кардиоваскулярных событий, как летальный исход, инфаркт миокарда (ИМ), ОНМК/ТИА, тромбоз в зоне реконструкции, кровотечение типа 3b и выше по шкале Bleeding Academic Research Consortium (BARC), комбинированная конечная точка (смерть + OHМК/ТИА + ИМ). Визуализация зоны реконструкции 
выполнялась посредством цветного дуплексного сканирования после операции. Длительность отдаленного пе-

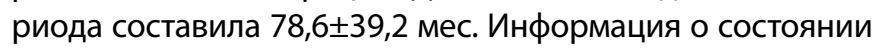
больных была получена путем телефонного анкетирования, вызова в клинику для прохождения скринингового обследования.

\section{Статистический анализ}

Определение типа распределения осуществлялось с помощью критерия Колмогорова-Смирнова. Сравнение групп проводили с применением критерия Краскела-Уоллиса и хи-квадрата Пирсона $\left(\mathrm{X}^{2}\right)$. При сравнении качественных показателей групп по парам применялся критерий $X^{2}$ с поправкой Йейтса. Графики выживаемости строились на основе анализа КапланаМейера. Сравнение графиков производилось при помощи Logrank test. Различия оценивались как значимые при $\mathrm{p}<0,05$. При наличии значимых различий между всеми группами $\left(p_{\text {общ }}\right)$ указывались конкретные группы, между которыми также выявлялось $p<0,05$. С целью выявления факторов риска развития осложнений была проведена бинарная логистическая регрессия с пошаговым включением и исключением предикторов (stepwise logistic regression). Результаты исследований обработаны при помощи пакета прикладных программ Graph Pad Prism (www.graphpad.com) и MedCalc 19.2.1 (www.medcalc.org).

\section{Этическая экспертиза}

Исследование выполнялось в соответствии со стандартами надлежащей клинической практики (Good Clinical Practice) и принципами Хельсинкской декларации. Все пациенты подписали согласие на реализованную стратегию лечения. Исследование не противоречит законам Российской Федерации.

\section{РЕЗУЛЬТАТЫ}

По клинико-анамнестическим показателям группы оказались не полностью сопоставимы. Ожидаемо больные СД2 были моложе, среди них чаще всего диагностировались хроническая почечная недостаточность, МФА, ожирение. Ввиду выраженной коморбидности показатель EuroSCORE II составил наибольшие значения именно в этой когорте пациентов (табл. 1).

По показателям эхокардиографии и коронарографии (с учетом, в том числе, резидуального SYNTAX score) группы были сопоставимы. По данным ангиографии каротидных артерий среди пациентов с СД2 чаще визуализировались двусторонние стенозы ВСА, нестабильная атеросклеротическая бляшка (табл. 2).

В госпитальном послеоперационном периоде пациенты были несопоставимы по показателю ОНМК/ТИА. Наибольшее количество ишемических инсультов было зафиксировано в группах эверсионной и классической КЭЭ среди больных с СД2. Такая тенденция привела к максимальным значениям комбинированной конечной точки в этих когортах (табл. 3).

На отдаленном этапе наблюдения пациенты с СД2 после классической техники операции продемонстрировали наибольшие показатели всех вариантов осложнений: смерть $(p<0,0001)$, ИМ $(p=0,011)$, ишемический инсульт
( $<<0,0001)$, рестеноз/окклюзия ВСА (p<0,0001), комбинированная конечная точка $(\mathrm{p}<0,0001)$.

При этом вторую позицию по распространенности неблагоприятных событий заняла группа эверсионной КЭЭ с нарушением углеводного обмена (табл. 4). Однако следует отметить, что по частоте развития всех осложнений между классической КЭЭ без СД2 и эверсионной КЭЭ с СД2 статистических различий получено не было. Такие обстоятельства демонстрируют, что имплантация заплаты, независимо от сопутствующих факторов, сопровождается повышенным риском развития не только ИМ, но и рестеноза, а также связанного с ним ишемического инсульта.

При анализе графиков выживаемости выявлена та же тенденция: группа классической КЭЭ с пластикой зоны реконструкции заплатой характеризуется наибольшими значениями всех видов осложнений (рис. 1-4). При этом только по показателю ИМ она сопоставима с результатами эверсионной техники. Такая особенность позволяет подтвердить заключение о том, что имплантация заплаты сопряжена с более высокой вероятностью развития ассоциированных с ней состояний - рестеноза, ишемического инсульта, летального исхода. Дополнительно необходимо отметить, что в группах классической КЭЭ подавляющее количество ОНМК/ТИА было зафиксировано в первый год наблюдения, что связано с развитием ранних рестенозов зоны ВСА в зоне реконструкции.

По результатам бинарной логистической регрессии с пошаговым включением и исключением предикторов были выявлены следующие факторы риска: СД2, требующий терапии инсулином; билатеральные стенозы ВСА; реализация классической КЭЭ с пластикой зоны реконструкции заплатой (табл. 5).

\section{ОБСУЖДЕНИЕ}

Сопоставление с другими публикациями

Первая статья в отечественной литературе, посвященная результатам КЭЭ при наличии СД, по данным электронной библиотеки elibrary.ru, была опубликована в 2008 г. А.И. Мешкаускене и соавт. [13]. На базе Вильнюсского университета (Литва) авторы проанализировали результаты операций 100 пациентов сСД и 607 - без него за период 1995-2005 гг. Было отмечено, что для больных с нарушением углеводного обмена более характерны молодой возраст $(p<0,05)$, ожирение $(p<0,001)$, билатеральные стенозы ВСА $(p<0,01)$, курение $(p<0,001)$ [13]. Среди них же чаще диагностировалось развитие ишемического инсульта и летального исхода в послеоперационном периоде (12\% против 3,4\%; р<0,001) [13]. Взаимосвязь между ростом числа ОНМК/ТИА и уровнем гликемии более 8 ммоль/л была также отмечена и в исследовании М.М. Танашян и соавт. [14]. Результаты нашей работы также продемонстрировали, что на госпитальном этапе наблюдения, независимо от вида КЭЭ, у пациентов с СД2 возрастает частота развития ишемических инсультов. Однако объяснить причину такой отрицательной динамики одной лишь тяжестью коморбидного фона невозможно. Изучая патофизиологические механизмы большей склонности больных СД к формированию церебральной ишемии при пережатии ВСА, О.В. Каменская и соавт. пришли к интересным выводам [15]. 


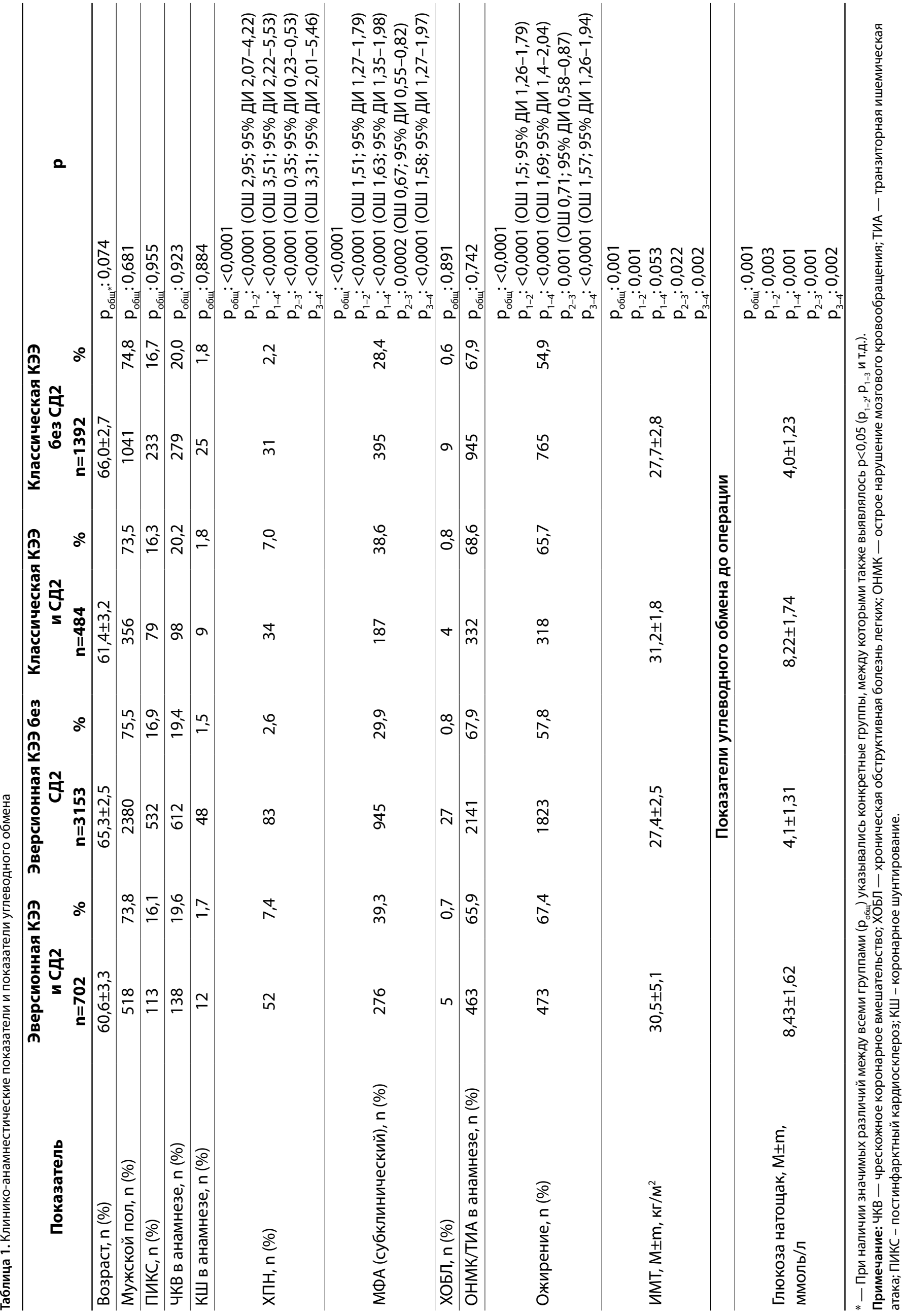




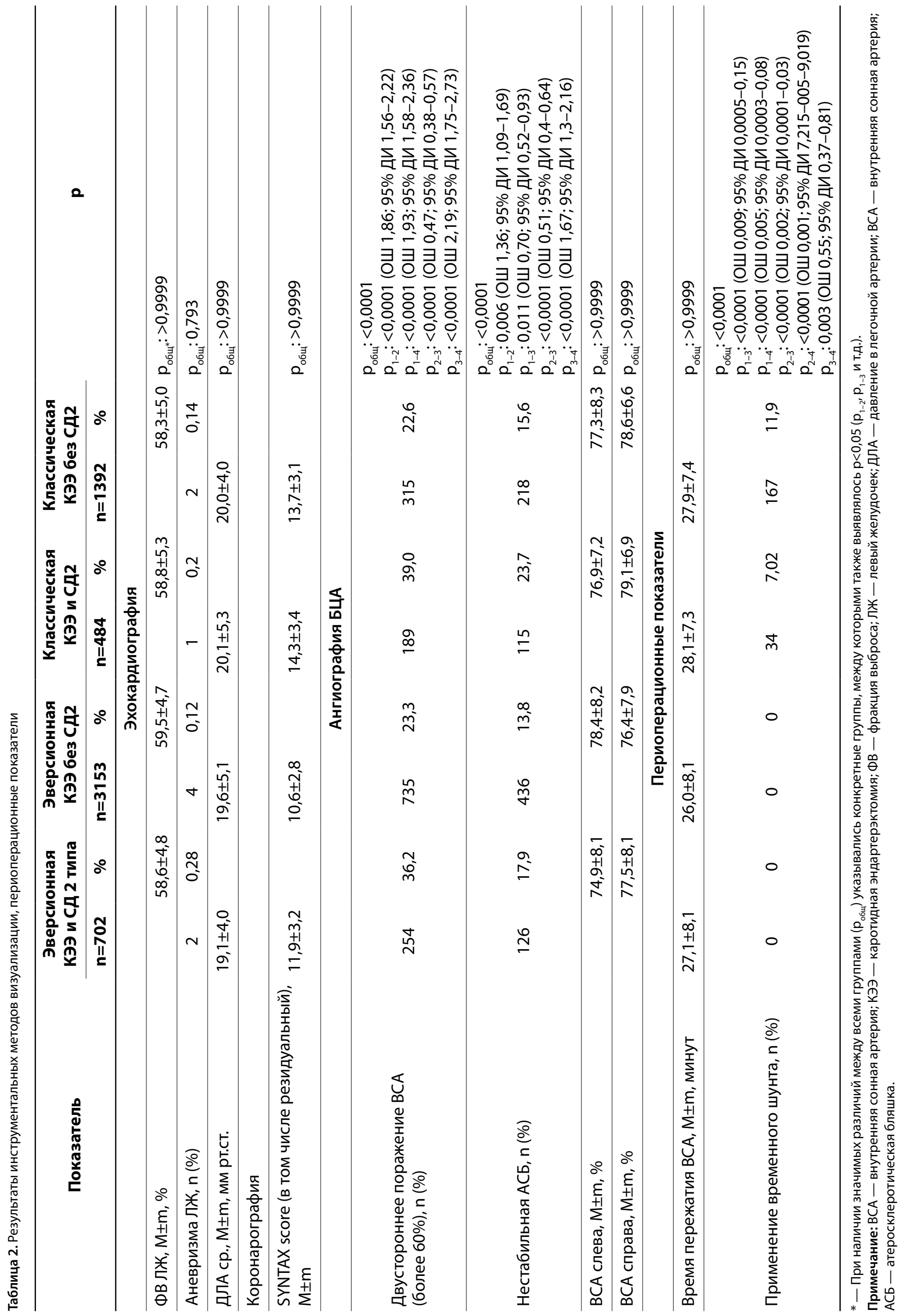




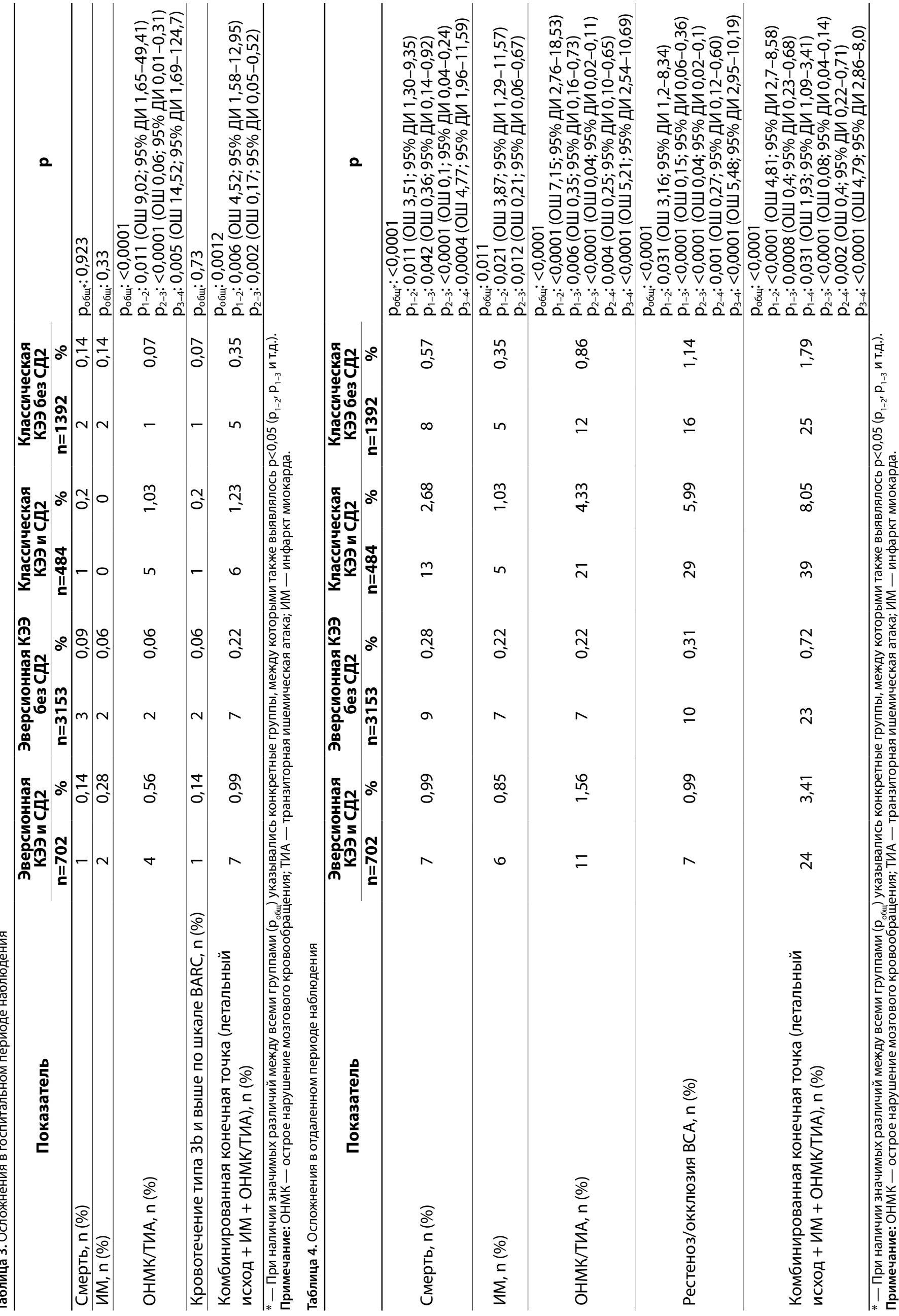




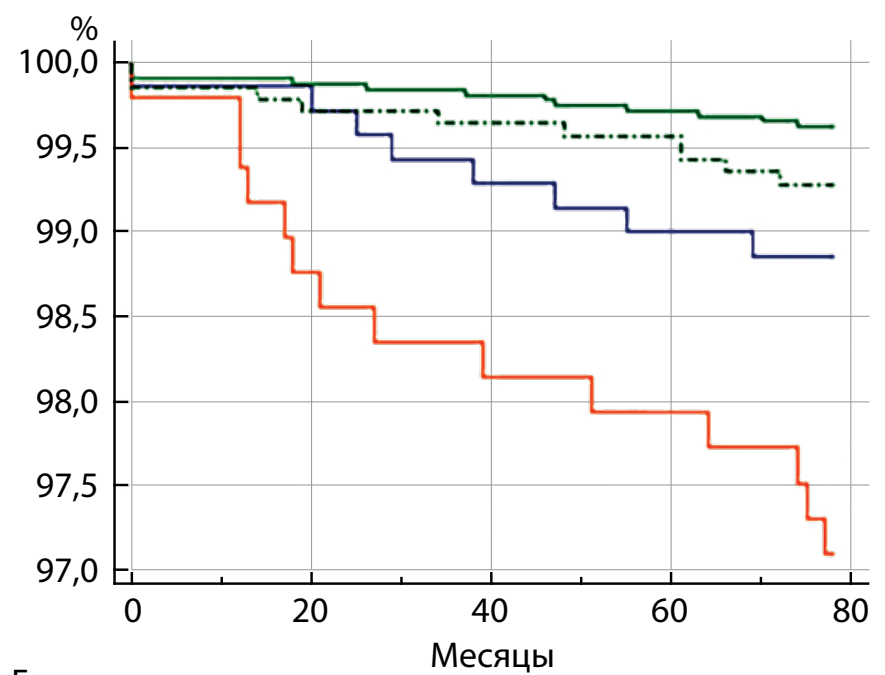

Группы

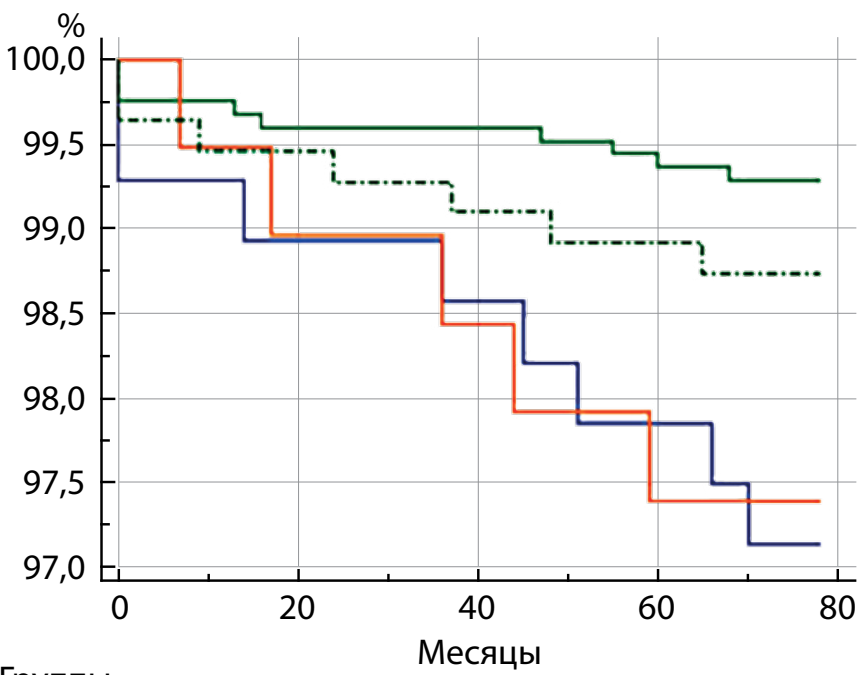

1

Группь

Месяцы

\begin{tabular}{r}
-1 \\
- \\
\hline \\
3 \\
-.... 4
\end{tabular}

Количество пациентов в зоне риска

\begin{tabular}{lccccc}
\hline Гр. 1 & 701 & 700 & 697 & 695 & 0 \\
Гр. 2 & 3150 & 3149 & 3147 & 3144 & 0 \\
Гр. 3 & 483 & 478 & 475 & 474 & 0 \\
Гр. 4 & 1390 & 1388 & 1387 & 1386 & 0 \\
\hline
\end{tabular}

Рисунок 1. Выживаемость, свободная от летального исхода (p<0,0001).

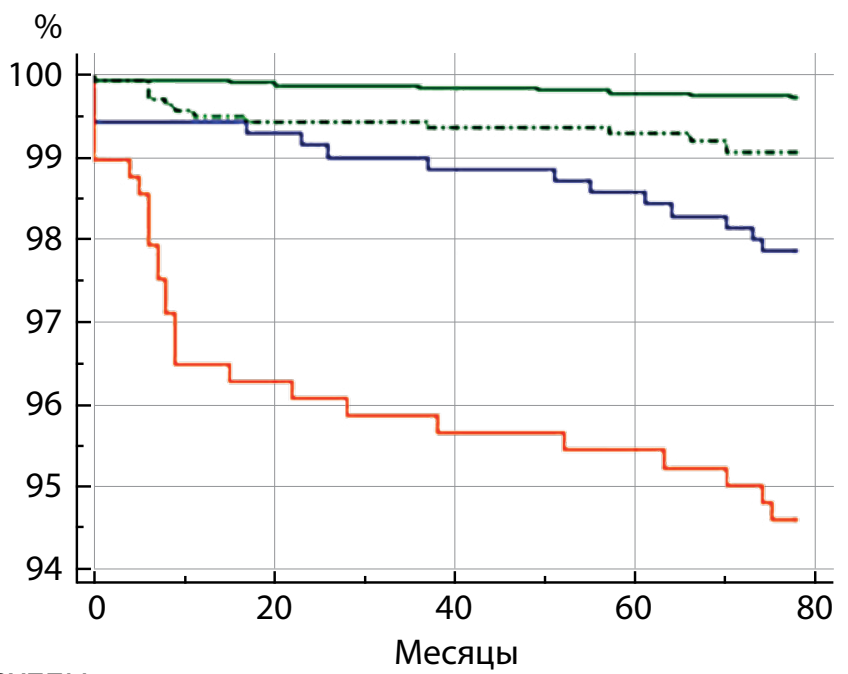

Группы

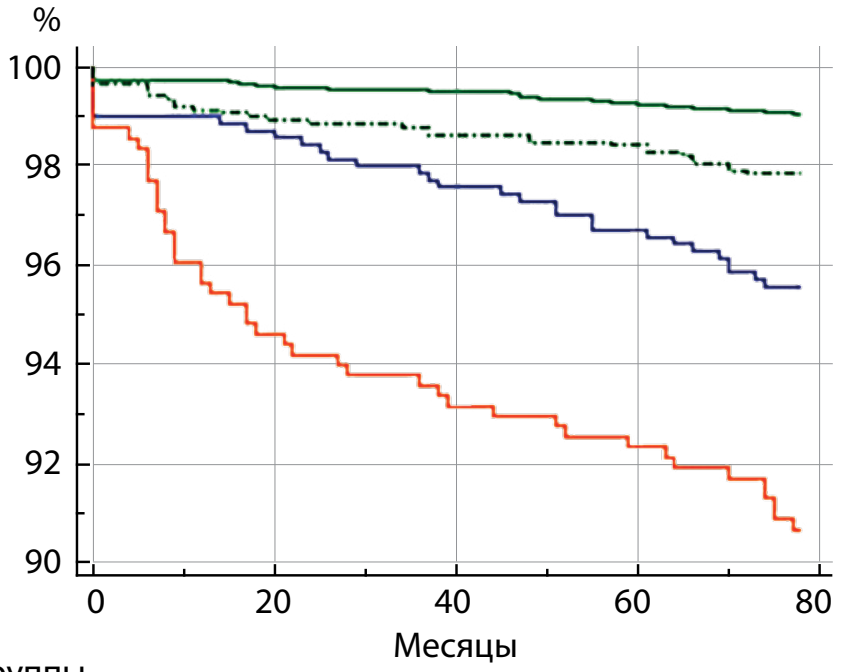

Группы

Количество пациентов в зоне риска

\begin{tabular}{lccccc}
\hline Гр. 1 & 700 & 699 & 698 & 696 & 0 \\
Гр. 2 & 3150 & 3148 & 3148 & 3145 & 0 \\
Гр. 3 & 484 & 480 & 477 & 474 & 0 \\
Гр. 4 & 1390 & 1389 & 1387 & 1386 & 0 \\
\hline
\end{tabular}

Рисунок 2. Выживаемость, свободная от инфаркта миокарда $(p=0,009)$.

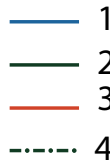

1
$-\quad 2$

3

-..... 4

\begin{tabular}{lccccc}
\hline \multicolumn{5}{c}{ Количество пациентов в зоне риска } \\
\hline Гр. 1 & 698 & 697 & 694 & 692 & 0 \\
Гр. 2 & 3151 & 3149 & 3148 & 3146 & 0 \\
Гр. 3 & 479 & 466 & 463 & 462 & 0 \\
Гр. 4 & 1391 & 1384 & 1383 & 1382 & 0 \\
\hline
\end{tabular}

Рисунок 3. Выживаемость, свободная от острого нарушения мозгового кровообращения/транзиторной ишемической атаки $(p<0,0001)$.

\begin{tabular}{lccccc}
\hline \multicolumn{5}{c}{ Количество пациентов в зоне риска } \\
\hline Гр. 1 & 695 & 692 & 685 & 679 & 0 \\
Гр. 2 & 3145 & 3140 & 3137 & 3129 & 0 \\
Гр. 3 & 478 & 458 & 451 & 447 & 0 \\
Гр. 4 & 1387 & 1377 & 1373 & 1370 & 0 \\
\hline
\end{tabular}

Рисунок 4. Выживаемость, свободная от комбинированной конечной точки $(p<0,0001)$. 
Таблица 5. Предикторы развития отдаленных осложнений в общей выборке больных

\begin{tabular}{|c|c|c|}
\hline \multicolumn{3}{|l|}{ ОШ и 95\% Ди } \\
\hline Переменная & ОШ & 95\% ди \\
\hline СД2, требующий терапии инсулином & 3,41 & $1,72-6,63$ \\
\hline Билатеральные стенозы ВСА & 3,61 & $1,82-7,58$ \\
\hline Реализация классической КЭЭ с пластикой зоны реконструкции заплатой & 2,23 & $1,02-5,88$ \\
\hline \multicolumn{3}{|l|}{ Анализ ROC-кривой } \\
\hline Площадь под ROC-кривой & \multicolumn{2}{|c|}{0,659} \\
\hline Стандартная ошибка & \multicolumn{2}{|c|}{0,0328} \\
\hline 95\% Ди & \multicolumn{2}{|c|}{$2,623-3,811$} \\
\hline
\end{tabular}

На основе анализа газового и электролитного состава крови авторы установили, что у этих пациентов отмечается возрастание экстракции кислорода из крови. Такая особенность свидетельствует о повышенных затратах резервов газотранспортной системы крови для адекватного обеспечения головного мозга [15]. В свою очередь, в условиях наличия двусторонних стенозов ВСА и удовлетворительных показателей ретроградного давления/ церебральной оксиметрии на фоне пережатия артерий и отсутствия показаний для установки ВШ может развиться гипоксия с последующими необратимыми последствиями [15]. В итоге эти больные подвергаются КЭЭ с большим риском развития церебральных осложнений. Таким образом, учитывая полученные данные, показания для установки ВШ в условиях нарушения углеводного обмена и билатеральных стенозов ВСА нужно рассматривать более прецизионно.

Говоря об отдаленных результатах КЭЭ в зависимости от наличия/отсутствия СД2, единственная отечественная работа, посвященная этому вопросу, была проведена под руководством Ю.И. Казакова и соавт. Авторы сообщили, что в группе больных с СД2 кумулятивная выживаемость через 5 лет составила 54,2士7,2\% относительно

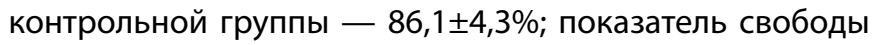

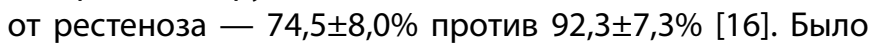
продемонстрировано, что предикторами летальных исходов являются возраст >65 лет, длительность СД >5 лет, уровень гликированного гемоглобина более 7,5\% [16].

В недавнем корейском исследовании авторы доказали, что высокий уровень гликемии, требующий терапии инсулином, сопряжен с ростом летальности в отдаленном послеоперационном периоде [17]. Эта закономерность объясняется в первую очередь тяжестью коморбидного фона, который сопровождает СД2, проявляющегося в формировании ангиопатии, поражении почек, сердца, печени и т.д. [17]. Таким образом, в своем исследовании авторы получили статистически значимые межгрупповые различия по показателям ОНMK $(p=0,006)$ и комбинированной конечной точки $(p=0,04)$, подтвердив, что СД2 является независимым фактором риска развития осложнений отдаленного периода [17].

A.B. Pothof и соавт. пришли к аналогичным выводам. Однако дизайн исследования несколько отличался. Пациенты были разделены на три группы: 1-я - без СД2; 2-я - с СД2, требующим инсулинотерапии; 3-я - с СД2, требующим приема оральных гипогликемических препаратов [18]. Авторы продемонстрировали, что в 30-дневном послеоперационном периоде в 1-й группе отмечает- ся статистически меньшая частота развития показателя ОНМК+смерть относительно 2-й группы (1,5\% против 3,4\%; $p<0,001)$ [18]. При этом значимых различий между 1-й и 3-й группами выявлено не было ввиду того, что пациенты с более мягким течением СД имеют более благоприятный коморбидный фон, что обеспечивает им сопоставимую стабильность послеоперационных результатов (1,5\% против 2,1\%; $p=0,1)[18]$.

M.A. Hussain и соавт. провели крупный метаанализ 1241 исследования за период с 1946-2015 гг., посвященного госпитальным и отдаленным исходам КЭЭ в зависимости от наличия СД2 [19]. По результатам работы авторы пришли к выводам, что реваскуляризация у больных СД2 сопряжена с более высоким риском следующих исходов: периоперационный инсульт, госпитальная смерть, смерть в отдаленном периоде наблюдения [19]. Итоги нашего исследования согласуются с данными мировой литературы. В отдаленном периоде наблюдения у пациентов с СД2 статистически значимо возрастает число всех неблагоприятных кардиоваскулярных событий. Предикторами этих событий стали СД2, требующий терапии инсулином, билатеральные стенозы ВСА, реализация классической КЭЭ с пластикой зоны реконструкции заплатой. Тяжелое течение СД2 всегда сопровождается выраженным коморбидным фоном, дающим негативный прогноз продолжительности и качества жизни пациента. И бесспорно, что больной, нуждающийся в инсулинотерапии, чаще всего имеет большую длительность СД, сопряженную с сопутствующими этой патологии состояниями, относительно пациентов, принимающих оральную гипогликемическую терапию [14, 16-18]. Поэтому наличие данного фактора будет свидетельствовать о повышенном риске развития сердечно-сосудистых осложнений после реализованной реваскуляризации.

Другим важным идентификатором негативного прогноза стало формирование двусторонних стенозов BCA. Такое состояние может отражать более агрессивное течение МФА с возможным включением в процесс коронарного и периферического русла [20]. Диффузное многососудистое поражение в литературе неоднократно рассматривалось как угрожающее состояние, даже несмотря на реализованную реваскуляризацию всех заинтересованных бассейнов [20]. А совокупное течение СД2 только усугубляет процесс развития рестенозов и прогрессирования атеросклероза с последующей окклюзией и/или тромбозом зоны реконструкции $[12,20]$. 
Возвращаясь к генезу каротидного рестеноза, следует отметить, что этот процесс является мультитаргетным. В литературе неоднократно упоминалось, что мощный вклад в развитие потери просвета сосуда вносят как наследственность, так и особенности образа жизни, наличие метаболического синдрома $[2,3,13]$. Поэтому сопутствующее течение СД2 приводит к более раннему формированию рестеноза [12]. С другой стороны, применение заплаты во время КЭЭ расширяет каротидную луковицу, провоцируя нарушение физических показателей гемоциркуляции в этой зоне [12]. Образование участков пристеночного застоя и турбулентного кровотока катализирует процесс гиперплазии неоинтимы, вызывая потерю просвета сосуда уже через полгода после реваскуляризации. На этом фоне СД2 только ускоряет негативный тренд, ведущий к необратимым изменениям с последующими нежелательными явлениями, что подтвердили данные нашей работы [12]. В группе больных СД2, которым реализовывалась классическая техника операции с пластикой зоны реконструкции заплатой, было идентифицировано наибольшее количество всех неблагоприятных кардиоваскулярных событий в отдаленном периоде наблюдения. Таким образом, данная техника операции является наименее предпочтительной в этой когорте пациентов.

\section{Выводы}

Необходимо отметить, что наша работа является первым крупным отечественным многоцентровым ретроспективным исследованием, посвященным результатам КЭЭ в зависимости от наличия/отсутствия СД2. Дефицит российских статей на эту тему демонстрирует низкий интерес сосудистых хирургов к влиянию нарушения углеводного обмена на непосредственные и отдаленные исходы реваскуляризации головного мозга. Таким образом, полученные нами результаты привлекут дополнительное внимание медицинского сообщества к проблеме долгосрочной курации и прецизионному ведению пациентов с СД2 и КЭЭ в анамнезе.
Ограничения исследования

Ретроспективный характер исследования. Отсутствие рандомизации.

\section{ЗАКЛЮЧЕНИЕ}

Пациенты с СД2 и КЭЭ в анамнезе подвержены повышенному риску развития ишемического инсульта на госпитальном этапе наблюдения и неблагоприятных кардиоваскулярных состояний в отдаленном послеоперационном периоде. Классическая техника операции с пластикой зоны реконструкции биологической заплатой является наименее предпочтительной для больных с СД2 ввиду высокого риска рестеноза уже через 6 мес после реваскуляризации.

\section{ДОПОЛНИТЕЛЬНАЯ ИНФОРМАЦИЯ}

Источники финансирования. Работа выполнена по инициативе авторов без привлечения финансирования.

Конфликт интересов. Авторы декларируют отсутствие явных и потенциальных конфликтов интересов, связанных с содержанием настоящей статьи.

Участие авторов. Казанцев А.Н. - концепция и дизайн, написание статьи; Виноградов Р.А. - анализ полученных данных; Чернявский М.А. - анализ полученных данных; Кравчук В.Н. - анализ полученных данных; Шматов Д.В. - анализ полученных данных; Сорокин А.А. - сбор и обработка материалов; Ерофеев А.А. - сбор и обработка материалов; Луценко В.А. - сбор и обработка материалов; Султанов Р.В. - сбор и обработка материалов; Шабаев А.Р. обзор литературы; Раджабов И.М. - сбор и обработка материалов; Багдавадзе Г.Ш. - сбор и обработка материалов; Заркуа Н.Э. - сбор и обработка материалов; Матусевич В.В. - сбор и обработка материалов; Вайман Е.Ф. - сбор и обработка материалов; Солобуев А.И. - сбор и обработка материалов; Артюхов С.В. - сбор и обработка материалов; Лидер Р.Ю. - сбор и обработка материалов; Порханов В.А. - концепция и дизайн; Хубулава Г.Г. - концепция и дизайн.

Все авторы одобрили финальную версию статьи перед публикацией, выразили согласие нести ответственность за все аспекты работы, подразумевающую надлежащее изучение и решение вопросов, связанных с точностью или добросовестностью любой части работы.

\section{СПИСОК ЛИТЕРАТУРЫ | REFERENCES}

1. Казанцев А.Н., Тарасов Р.С., Бурков Н.Н., и др. Прогрессирование прецеребрального атеросклероза и предикторы ишемических осложнений у пациентов кардиохирургического профиля // Хирургия. Журнал им. Н.И. Пирогова. - 2020. — №7. - С. 31-38. [Kazantsev AN, Tarasov RS, Burkov NN, et al. Progression of precerebral atherosclerosis and predictors of ischemic complications in patients with cardiac surgery. Surgery. 2020;7:31-38. (In Russ.)]. doi: https://doi.org/10.17116/hirurgia202007131

2. Грачева С.А., Клефортова И.И., Шамхалова М.Ш. Распространенность сочетанного атеросклеротического поражения сосудов у больных сахарным диабетом // Сахарный диабет. - 2012. - T. 15. — №1. - C. 49-55. [Gracheva SA, Klefortova II, Shamkhalova MSh. Prevalence of combined atherosclerotic vascular lesions in patients with diabetes mellitus. Diabetes mellitus. 2012;15(1):49-55. (In Russ.)] doi: https://doi.org/10.14341/2072-0351-5979

3. Танашян М.М., Антонова К.В., Медведев Р.Б., и др. Симптомная и асимптомная ишемия головного мозга (по данным МРТ) у больных сахарным диабетом 2 типа после каротидной реваскуляризации // Сахарный диабет. — 2019. - Т. 22. — №1. - С. 14-24.
[Tanashyan MM, Antonova KV, Medvedev RB, et al. Symptomatic and asymptomatic cerebral ischemia (according to MRI data) in patients with type 2 diabetes mellitus after carotid revascularization. Diabetes mellitus. 2019;22(1):14-24. (In Russ.)]. doi: https://doi.org/10.14341/DM9633

4. Казанцев А.Н., Тарасов Р.С., Бурков Н.Н., и др. Госпитальные результаты чрескожного коронарного вмешательства и каротидной эндартерэктомии в гибридном и поэтапном режимах // Ангиология и сосудистая хирургия. — 2019. T. 25. - №1. - C. 101-107. [Kazantsev AN, Tarasov RS, Burkov NN, et al. Hospital results of percutaneous coronary intervention and carotid endarterectomy in hybrid and staged modes. Angiology and Vascular Surgery. 2019;25(1):101-107 (In Russ.)]. doi: https://doi.org/10.33529/angio2019114

5. Виноградов Р.А., Пыхтеев В.С., Мартиросова К.И., Лашевич К.А Прогнозирование периоперационных осложнений при каротидной эндартерэктомии // Хирургия. Журнал им. Н.И. Пирогова. - 2018. - №1. - C. 82-85. [Vinogradov RA, Pykhteev VS, Martirosova KI, Lashevich KA. Prediction of perioperative complications in carotid endarterectomy. Surgery. 2018;(1):82-85. (In Russ.)]. doi: https://doi.org/10.17116/hirurgia2018182-85 
6. Казанцев А.Н., Черных К.П., Лидер Р.Ю., и др. Гломус-сберегающая каротидная эндартерэктомия по А.Н. Казанцеву. Госпитальные и среднеотдаленные результаты // Патология кровообращения и кардиохирургия. - 2020. - T. 24. - №3. - C. 70-79. [Kazantsev AN, Chernykh KP, Leader RYu, et al. Glomus-sparing carotid endarterectomy according to AN Kazantsev. Hospital and mid-term results. Circulatory pathology and cardiac surgery. 2020;24(3):70-79. (In Russ.)] doi: https://doi.org/10.21688/1681-3472-2020-3-70-79

7. Гавриленко А.В., Куклин А.В., Аль-Юсеф Н.Н., и др. Метаанализ результатов эверсионной каротидной эндартерэктомии и эндартерэктомии с пластикой заплатой // Ангиология и сосудистая хирургия. - 2020. — Т. 26. — №1. — C. 176-183. [Gavrilenko AV, Kuklin AV, Al-Yusef NN, et al. Meta-analysis of the results of eversion carotid endarterectomy and endarterectomy with plastic patch. Angiology and vascular surgery. 2020;26(1):176-183. (In Russ.)]. doi: https://doi.org/10.33529/ANGIO2020121

8. Казанцев А.Н., Черных К.П., Заркуа Н.Э., и др. Новый способ гломуссберегающей каротидной эндартерэктомии по А.Н. Казанцеву: отсечение внутренней сонной артерии на площадке из наружной и общей сонной артерии // Российский кардиологический журнал. - 2020. - T. 25. - №8. - C. 10-17. [Kazantsev AN, Chernykh KP, Zarkua NE, et al. A new method of glomus-sparing carotid endarterectomy according to AN Kazantsev: cutting off the internal carotid artery at the site from the external and common carotid artery. Russian journal of cardiology. 2020;25(8):10-17. (In Russ.)]. doi: https://doi.org/10.15829/1560-4071-2020-3851

9. Шмырев В.И., Носенко Е.М., Романова М.С., и др. Клинические и ультразвуковые маркеры осложнений каротидной эндартерэктомии в послеоперационном периоде у больных с сосудистой мозговой недостаточностью // Хирургия. Журнал им. Н.И. Пирогова. - 2015. - №2. - C. 13-24. [Shmyrev VI, Nosenko EM, Romanova MS, et al. Clinical and ultrasound markers of complications of carotid endarterectomy in the postoperative period in patients with cerebrovascular insufficiency. Surgery. 2015;2:13-24. (In Russ.)]. doi: https://doi.org/10.17116/hirurgia2015213-24

10. Зыбин А.В., Покровский А.В., Тимина И.Е., и др. Оценка отдаленных результатов каротидной эндартерэктомии у пациентов на фоне терапии статинами // Диагностическая и интервенционная радиология. - 2018. - Т. 12. - № 3. - C. 20-28. [Zybin AV Pokrovsky AV, Timina IE, et al. Assessment of long-term results of carotid endarterectomy in patients treated with statins. Diagnostic and interventional radiology. 2018;12(3):20-28. (In Russ.)]. doi: https://doi.org/10.25512/DIR.2018.12.3.02

11. Казанцев А.Н., Бурков Н.Н., Борисов В.Г., и др. Компьютерное моделирование гемодинамических показателей в бифуркации сонных артерий после каротидной эндартерэктомии // Ангиология и сосудистая хирургия. - 2019. - Т. 25. - №3. - С. 107-112. [Kazantsev AN, Burkov NN, Borisov VG, et al. Computer modeling of hemodynamic parameters in the bifurcation of the carotid arteries after carotid endarterectomy. Angiology and Vascular Surgery. 2019;25(3):107-112. (In Russ.)]. doi: https://doi.org/10.33529/ANGIO2019311

12. Казанцев А.Н., Богомолова А.В., Бурков Н.Н., и др. Морфология рестеноза после классической каротидной эндартерэктомии с применением заплаты из диэпоксиобработанного ксеноперикарда // Кардиология и сердечно-сосудистая хирургия. 2020. - T. 13. — №1. — C. 68-71. [Kazantsev AN, Bogomolova AV, Burkov NN, et al. Morphology of restenosis after classical carotid endarterectomy using a patch made of diepoxy-treated xenopericardium. Cardiology and cardiovascular surgery. 2020;13(1):68-71. (In Russ.)]. doi: https://doi.org/10.17116/kardio202013011168
13. Мешкаускене А.И., Баркаускас Е.М., Гайгалайте В.Б. Взаимосвязь сахарного диабета с другими сердечно-сосудистыми факторами риска у больных стенозом внутренней сонной артерии // Терапевтический архив. - 2008. - Т. 80. — №1. - C. 49-52. [Meshkauskene Al, Barkauskas EM, Gaigalaite VB. The relationship of diabetes mellitus with other cardiovascular risk factors in patients with stenosis of the internal carotid artery. Therapeutic archive. 2008;80(1):49-52. (In Russ.)]

14. Танашян М.М., Скрылев С.И., Антонова К.В., Медведев Р.Б. Каротидная реваскуляризация у больных сахарным диабетом 2 типа. Значение хронической гипергликемии // Ангиология и сосудистая хирургия. - 2017. - Т. 23. — №4. - С. 99-106. [Tanashyan MM, Skrylev SI, Antonova KV, Medvedev RB. Carotid revascularization in patients with type 2 diabetes mellitus. The value of chronic hyperglycemia. Angiology and Vascular Surgery. 2017;23(4):99-106. (In Russ.)]

15. Каменская О.В., Левичева Е.Н., Логинова И.Ю., Карпенко А.А. Патофизиологические аспекты энергетического обмена головного мозга при сочетании системного атеросклероза и сахарного диабета 2-го типа // Кардиология и сердечнососудистая хирургия. — 2012. - Т. 5. — №1. — C. 60-63. [Kamenskaya OV, Levicheva EN, Loginova IYu, Karpenko AA Pathophysiological aspects of the energy metabolism of the brain in the combination of systemic atherosclerosis and type 2 diabetes mellitus. Cardiology and cardiovascular surgery. 2012;5(1):60-63. (In Russ.)]

16. Казаков Ю.И., Касьяненко А.П., Соколова Н.Ю., и др. Отдаленные результаты каротидной эндартерэктомии у больных с сахарным диабетом II типа // Ангиология и сосудистая хирургия. 2017. - T. 23. — №2. - C. 98-106. [Kazakov Yul, Kasianenko AP, Sokolova NYu., et al. Long-term results of carotid endarterectomy in patients with type II diabetes mellitus. Angiology and vascular surgery. 2017;23(2):98-106. (In Russ.)]

17. Jeong MJ, Kwon $\mathrm{H}$, Jung $\mathrm{CH}$, et al. Comparison of outcomes after carotid endarterectomy between type 2 diabetic and non-diabetic patients with significant carotid stenosis. Cardiovasc Diabetol. 2019;18(1):41. doi: https://doi.org/10.1186/s12933-019-0848-7

18. Pothof $A B, O$ 'Donnell TFX, Swerdlow NJ, et al. Risk of insulindependent diabetes mellitus in patients undergoing carotid endarterectomy. J Vasc Surg. 2019;69(3):814-823. doi: https://doi.org/10.1016/j.jvs.2018.05.250.

19. Назаренко М.С., Марков А.В., Королева Ю.А., и др. Идентификация дифференциально метилированных генов, потенциально связанных с атеросклерозом у человека // Российский кардиологический журнал. - 2017. - Т. 22. №10. - C. 42-48. [Nazarenko MS, Markov AV, Koroleva YuA, et al. Identification of differentially methylated genes potentially associated with atherosclerosis in humans. Russian journal of cardiology. 2017;22(10):42-48. (In Russ.)]. doi: https://doi.org/10.15829/1560-4071-2017-10-42-48

20. Тарасов Р.С., Казанцев А.Н., Иванов С.В., и др. Хирургическое лечение мультифокального атеросклероза: патология коронарного и брахиоцефального бассейнов и предикторы развития ранних неблагоприятных событий // Кардиоваскулярная терапия и профилактика. - 2017. - Т. 16. — №4. - С. 37-44. [Tarasov RS, Kazantsev AN, Ivanov SV, et al. Surgical treatment of multifocal atherosclerosis: pathology of the coronary and brachiocephalic basins and predictors of early adverse events. Cardiovascular therapy and prevention. 2017;16(4):37-44. (In Russ.)] doi: https://doi.org/10.15829/1728-8800-2017-4-37-44

\section{ИНФОРМАЦИЯ ОБ АВТОРАХ [AUTHORS INFO]}

*Казанцев Антон Николаевич, сердечно-сосудистый хирург [Anton N. Kazantsev, cardiovascular surgeon]; aдpec: Россия, 193312, Санкт-Петербург, пр. Солидарности, д. 4 [address: 4 Avenue of Solidarity, 193312 St. Petersburg, Russia]; ORCID: https://orcid.org/0000-0002-1115-609X; eLibrary SPIN: 8396-1845; e-mail: dr.antonio.kazantsev@mail.ru

Виноградов Роман Александрович, д.м.н., доцент [Roman A. Vinogradov, MD, PhD, Associate Professor]; ORCID: https://orcid.org/0000-0001-9421-586X; eLibrary SPIN: 7211-3229; e-mail: viromal@mail.ru Чернявский Михаил Александрович, д.м.н., гл.н.с. [Michael A. Chernyavsky, MD, PhD, chief research associate]; ORCID: https://orcid.org/0000-0003-1214-0150; eLibrary SPIN: 5009-7818; e-mail: machern@mail.ru Кравчук Вячеслав Николаевич, д.м.Н., профессор [Vyacheslav N. Kravchuk, MD, PhD, Professor]; ORCID: https://orcid.org/0000-0002-6337-104X; eLibrary SPIN: 4227-2846; e-mail: kravchuk9@yandex.ru 
Шматов Дмитрий Викторович, д.м.н., профессор [Dmitriy V. Shmatov, MD, PhD, Professor]; ORCID: https://orcid.org/0000-0002-1296-8161; eLibrary SPIN: 4242-2812; e-mail: dv.shmatov@gmail.com Сорокин Андрей Александрович, сердечно-сосудистый хирург [Andrey A. Sorokin, cardiovascular surgeon]; ORCID: https://orcid.org/0000-0003-0493-4209; eLibrary SPIN: 3463-1021; e-mail: sorokin@gmail.com

Ерофеев Александр Алексеевмч, к.м.н., зам. главного врача по хирургии, полковник медицинской службы [Alexandr A. Erofeev, Ph.D., Deputy Chief Physician for Surgery, Colonel of the Medical Service]; ORCID: https://orcid.org/ 0000-0003-3814-9831; eLibrary SPIN: 4321-2821; e-mail: aerofeev1963@gmail.com Луценко Виктор Анатольевич, к.м.н., сердечно-сосудистый хирург [Victor A. Lutsenko, MD, PhD, cardiovascular surgeon]; ORCID: https://orcid.org/0000-0003-3188-2790; eLibrary SPIN: 4251-2281; e-mail: aaapppmmmooo@gmail.com Султанов Роман Владимирович, к.M.н. [Roman V. Sultanov, MD, PhD]; ORCID: https://orcid.org/0000-0003-2888-1797; eLibrary SPIN: 1382-1295; e-mail: aaapppmmmooo@gmail.com

Шабаев Амин Рашитович, сердечно-сосудистый хирург [Amin R. Shabaev, cardiovascular surgeon]; ORCID: https://orcid.org/0000-0002-9734-8462; eLibrary SPIN: 6119-0504; e-mail: neirohirurgi@yandex.ru Раджабов Ислам Магомедович, зав. отделением сосудистой хирургии [Islam M. Radjabov, Head department of vascular surgery]; ORCID: https://orcid.org/0000-0002-7915-1615; eLibrary SPIN: 2075-2131; e-mail: aaapppmmmooo@rambler.ru

Багдавадзе Годерзи Шотаевич, ординатор [Goderzi Sh. Bagdavadze, clinical resident]; ORCID: https://orcid.org/0000-0001-5970-6209; eLibrary SPIN: 2801-9655; e-mail: gud_777@bk.ru

Заркуа Нонна Энриковна, д.м.н., доцент [Nonna E. Zarkua, MD, PhD, Associate Professor]; ORCID: https://orcid.org/0000-0002-7457-3149; eLibrary SPIN: 1319-2534; e-mail: tatazarkua@mail.ru

Матусевич Вячеслав Викторович, сердечно-сосудистый хирург [Vyacheslav V. Matusevich, cardiovascular surgeon]; ORCID: https://orcid.org/0000-0001-9461-2726; eLibrary SPIN: 1139-0513; e-mail: dr.matusevich@mail.ru

Вайман Евгений Федорович, к.М.н., доцент [Evgeniy F. Vaiman, MD, PhD, Associate Professor]; ORCID: https://orcid.org/0000-0001-5784-5029; eLibrary SPIN: 3124-0413; e-mail: evgeny1962@mail.ru

Солобуев Алексей Игоревич, ассистент кафедры [Aleksey I. Solobuev, assistant of the department]; ORCID: https://orcid.org/0000-0003-2832-662X; eLibrary SPIN: 2859-1096; e-mail: aaapppmmmooo@rambler.ru

Артюхов Сергей Викторович, к.м.H. [Sergey V. Artyukhov, MD, PhD]; ORCID: https://orcid.org/0000-0001-8249-3790; eLibrary SPIN: 1894-6265; e-mail: art_serg@mail.ru

Лидер Роман Юрьевич, студент [Roman Yu. Lider, student]; ORCID: https://orcid.org/0000-0002-3844-2715; eLibrary SPIN: 3723-4648; e-mail: aaapppmmmooo@rambler.ru

Порханов Владимир Алексеевич, Д.М.Н., профессор, академик PAH [Vladimir A. Porkhanov, MD, PhD, Professor, Academician of the Russian Academy of Sciences]; ORCID: https://orcid.org/0000-0003-0572-1395; eLibrary SPIN: 2446-5933; e-mail: viromal@mail.ru

Хубулава Геннадий Григорьевич, д.м.Н., профессор, академик PAH [Gennadiy G. Khubulava, MD, PhD, Professor, Academician of the Russian Academy of Sciences]; ORCID: https://orcid.org/ 0000-0002-9242-994; eLibrary SPIN: 1007-8730; e-mail: viromal@mail.ru

\section{ЦИТИРОВАТЬ:}

Казанцев А.Н., Виноградов Р.А., Чернявский М.А., Кравчук В.Н., Шматов Д.В., Сорокин А.А., Ерофеев А.А., Луценко В.А., Султанов Р.В., Шабаев А.Р., Раджабов И.М., Баздавадзе Г.Ш., Заркуа Н.Э., Матусевич В.В., Вайман Е.Ф., Солобуев А.И., Артюхов С.В., Лидер Р.Ю., Порханов В.А., Хубулава Г.Г. Прогнозирование ремиссии сахарного диабета 2 типа после выполнения бариатрической операции // Сахарный диабет. — 2021. — Т. 24. — №6. — С. $536-547$. doi: https://doi.org/10.14341/DM12722

\section{TO CITE THIS ARTICLE:}

Kazantsev AN, Vinogradov RA, Chernyavsky MA, Kravchuk VN, Shmatov DV, Sorokin AA, Erofeev AA, Lutsenko VA, Sultanov RV, Shabaev AR, Radjabov IM, Bagdavadze GSh, Zarkua NE, Matusevich VV, Vaiman EF, Solobuev Al, Artyukhov SV, Lider RYu, Porkhanov VA, Khubulava GG. Results of different kinds of carotid endarterectomy in patients with and without type 2 diabetes mellitus. Diabetes Mellitus. 2021;24(6):536-547. doi: https://doi.org/10.14341/DM12722 